ARTICLE

Received 28 Jan 2015 | Accepted 1 May 2015 | Published 11 Jun $2015 \quad$ DOl: 10.1038/ncomms8391 OPEN

\title{
Somatic CRISPR/Cas9-mediated tumour suppressor disruption enables versatile brain tumour modelling
}

Marc Zuckermann ${ }^{1}$, Volker Hovestadt ${ }^{1}$, Christiane B. Knobbe-Thomsen ${ }^{2,3}$, Marc Zapatka', Paul A. Northcott ${ }^{4}$, Kathrin Schramm 1 , Jelena Belic ${ }^{1}$, David T.W. Jones ${ }^{4}$, Barbara Tschida ${ }^{5}$, Branden Moriarity ${ }^{5}$, David Largaespada ${ }^{5}$, Martine F. Roussel ${ }^{6}$, Andrey Korshunov7,8, Guido Reifenberger ${ }^{2,3}$, Stefan M. Pfister ${ }^{4}$, Peter Lichter ${ }^{1}$, Daisuke Kawauchi ${ }^{4} \&$ Jan Gronych ${ }^{1}$

In vivo functional investigation of oncogenes using somatic gene transfer has been successfully exploited to validate their role in tumorigenesis. For tumour suppressor genes this has proven more challenging due to technical aspects. To provide a flexible and effective method for investigating somatic loss-of-function alterations and their influence on tumorigenesis, we have established CRISPR/Cas9-mediated somatic gene disruption, allowing for in vivo targeting of TSGs. Here we demonstrate the utility of this approach by deleting single (Ptch1) or multiple genes (Trp53, Pten, Nf1) in the mouse brain, resulting in the development of medulloblastoma and glioblastoma, respectively. Using whole-genome sequencing (WGS) we characterized the medulloblastoma-driving Ptch1 deletions in detail and show that no off-targets were detected in these tumours. This method provides a fast and convenient system for validating the emerging wealth of novel candidate tumour suppressor genes and the generation of faithful animal models of human cancer.

\footnotetext{
${ }^{1}$ Division of Molecular Genetics, German Cancer Research Center (DKFZ), Im Neuenheimer Feld 280, 69120 Heidelberg, Germany. ${ }^{2}$ Department of Neuropathology, Heinrich Heine University Düsseldorf, Moorenstrasse 5, 40225 Düsseldorf, Germany. ${ }^{3}$ German Cancer Consortium (DKTK), partner site Essen/Düsseldorf, 40225 Düsseldorf, Germany. ${ }^{4}$ Division of Pediatric Neurooncology, German Cancer Research Center (DKFZ), Im Neuenheimer Feld 280, 69120 Heidelberg, Germany. ${ }^{5}$ Masonic Cancer Center, University of Minnesota, Minneapolis, Minnesota 55455, USA. ${ }^{6}$ Tumor Cell Biology, St Jude Children's Research Hospital, 262 Danny Thomas Place, Memphis, Tennessee 38105, USA. ${ }^{7}$ Department of Neuropathology, University of Heidelberg, Im Neuenheimer Feld 220, 69120 Heidelberg, Germany. ${ }^{8}$ Clinical Cooperation Unit Neuropathology, German Cancer Research Center (DKFZ), Im Neuenheimer Feld 220-221, 69120 Heidelberg, Germany. Correspondence and requests for materials should be addressed to D.K. (email: d.kawauchi@dkfz.de) or to J.G. (email: j.gronych@dkfz.de).
} 
$\mathrm{M}$ odelling cancer in mice through engineering of candidate genes in the germline has long been the gold standard for the validation of putative oncogenes or tumour suppressor genes (TSGs). For TSGs, whereby loss-offunction (LOF) mutations act as a driver for malignant transformation, this has traditionally been accomplished using constitutive or cell-type-specific knockout strategies mediated by homologous recombination in embryonic stem cells. Although this allows evaluation of cell-type-specific susceptibility to malignant transformation, generation of genetically engineered mouse models (GEMMs) is a time-consuming process. For in vivo investigation of a large number of molecular alterations, such as the many new candidates currently emerging from largescale tumour genome-sequencing efforts ${ }^{1}$, a faster and more flexible method is required. We therefore sought to adapt the CRISPR/Cas9-guided endonuclease technique ${ }^{2-4}$ for the somatic disruption of candidate TSGs and thereby complement aforementioned already existing models like GEMM. The locusspecific cutting by the Cas 9 nuclease is mediated by a guide-RNA (gRNA) encoded on the same vector, targeting a 20mer cognate sequence in the genome. Upon introduction of a double-strand break, erroneous non-homologous end-joining (NHEJ) repair evokes insertions/deletions (indels) and potential frameshift mutations, typically resulting in LOF of the target gene ${ }^{5}$.

While there have been reports of cancer modelling using somatic transfer of CRISPR/Cas9 to the lung by viral-mediated transduction $^{6}$ or to the liver by hydrodynamic injection ${ }^{7}$, here we aimed at using somatic gene transfer of CRISPR/Cas9 to induce brain tumours. We therefore applied polyethylenimine (PEI)-mediated transfection ${ }^{8}$ or in utero electroporation of the developing brain ${ }^{9}$. These techniques do not suffer from size limitations applying to viral vectors, are mouse strain independent, provide tissue specificity and yet can be adapted to target a variety of different mouse tissues. We further sought to use these systems to induce specific types of brain tumours.

As a model system, we first focused on sonic hedgehog $(\mathrm{SHH})$ medulloblastoma $(\mathrm{MB})^{10}$, a malignant childhood brain tumour ${ }^{11}$. Genomic studies have delineated four distinct MB subgroups, each with characteristic molecular and clinical features ${ }^{10,12}$. SHH subgroup MBs originate from granule neuron precursors (GNPs), a cell type that is exclusively present in the developing cerebellum, and whose proliferation is regulated by the $\mathrm{SHH}$ pathway ${ }^{13}$. Germline mouse models that harbour heterozygous deletion of the Ptch 1 gene (i.e., Ptch $1^{+/-}$), encoding an essential negative regulator of the SHH pathway, develop aberrant clusters of GNPs, so-called 'preneoplastic hyperplasia'. In 14-20\% of these animals, these clusters continue malignant transformation and form full-blown $\mathrm{SHH} \mathrm{MB}$ within 3 months ${ }^{14,15}$. The efficiency of tumor development can be increased by inducing conditional homozygous Ptch1 deletion in relevant stem/progenitor cell populations ${ }^{16,17}$ or by breeding Ptch $1^{+/-}$mice to a Trp53-null background ${ }^{18}$, which is also seen in a proportion of patients with highly aggressive human $\mathrm{SHH} \mathrm{MB}{ }^{19}$. Based on this data, we aimed to somatically disrupt Ptch1 using in vivo transfection (P0) and in utero electroporation (E13.5) of plasmids encoding both the Cas9 nuclease and a gRNA targeting the endogenous Ptch1 locus. Using these approaches we were able to induce tumours resembling $\mathrm{MB}$ with a high penetrance. Next generation sequencing of these tumours showed large on-site deletions disrupting the Ptch1 locus whereas no off-target could be detected.

Furthermore, we sought to extend the utility of this approach to glioblastoma (GBM), the most aggressive primary brain tumor $^{20}$. These astrocytic tumours originate from neural stem or glial progenitor cells primarily in the forebrain ${ }^{21,22}$ and frequently harbour mutations in the key TSGs P53, PTEN and
NF1 (refs 23,24). Previously, it was shown that $h G F A P-C r e ; N f 1^{f /+}$; Trp53 $^{f / f} ;$ Pten $^{f /+}$ mice develop tumours resembling human glioblastoma with high penetrance ${ }^{25,26}$. To model these tumours somatically with the CRISPR/Cas9 system, we used in utero electroporation of the developing prosencephalon ${ }^{9}$. By delivering three plasmids simultaneously, encoding Cas9 together with gRNAs targeting Nf1, Trp53 and Pten, we were able to induce highly aggressive tumours, resembling human GBMs in all of the animals (Fig. 1a).

Collectively, in this study we show that the CRISPR/Cas system can be used to somatically induce LOF mutations in the murine brain leading to the induction of various, specific types of brain tumours with high penetrance. This approach will foster the in vivo validation of TSGs identified in recent next generation sequencing studies.

\section{Results}

Postnatal somatic CRISPR/Cas-mediated deletion of Ptch1. We first tested a set of vectors (pX330) carrying Cas 9 and gRNAs targeting Ptch1 in vitro using NIH3T3 cells. The two most effective gRNAs as assessed by SURVEYOR assays (designated Ptch1.1 and Ptch1.3, targeting the second exon of Ptch1; Supplementary Fig. 1) were used for further in vivo analysis. For postnatal delivery of pX330 plasmids targeting Ptch1 to GNPs, we applied PEI-mediated in vivo transfection by stereotactic injection into the neonatal cerebellum (P0) of wild-type (WT) mice. Successful targeting of the cerebellum was confirmed by trypan blue injection (Supplementary Fig. 2a). Expression of transfected plasmids was assessed by co-transfection of a luciferase-encoding plasmid and subsequent bioluminescence imaging (Supplementary Fig. 2b). We confirmed successful indel formation centred around the gPtch1.1 site in vivo at postnatal day P7 by targeted deep sequencing (Supplementary Fig. 3). Despite successful disruption of the Ptch1 TSG, none of these animals developed $\mathrm{MB}$ within a period of 6 months (Table 1). Histologic analysis of brains 8 weeks after birth, however, revealed a small neoplastic lesion characterized by aberrant accumulation of GNPlike cells on the surface of the cerebellum that was restricted to a single folium (Fig. 1b; Table 1). This is consistent with previously published data in which Ptch1 germline deletion leads to the induction of 'preneoplastic lesions' visible 3-6 weeks after birth ${ }^{27}$. These cells were still proliferating, as indicated by immunopositivity for the proliferation marker PCNA (Supplementary Fig. 4).

Germline deletion of the Trp53 tumour suppressor locus increases the efficiency of $\mathrm{SHH} \mathrm{MB}$ induction in mice ${ }^{18}$. We therefore PEI-transfected gPtch1.1/Cas 9 or gPtch1.3/Cas 9 into the developing cerebellum (P0) of $\operatorname{Trp} 53^{+} \mathcal{P}_{-}$and $\operatorname{Trp} 53^{-/-}$ animals. One of the $\operatorname{Trp} 53^{+/-}$mice showed a small neoplastic lesion similar to the lesion we found in the WT animal $(n=1 / 5)$. The Trp53-/- animals, however, started to develop neurological symptoms after $\sim 5$ weeks (Supplementary Fig. 5). Post mortem histological assessment revealed presence of tumours resembling the histology of human MB (Fig. 1c). In total we observed tumours in 8/10 animals (Table 1). As controls, we used the pX330 plasmid encoding a non-target gRNA (gNT) together with Cas9. None of these animals developed any pre-neoplasia or tumours (Table 1). This demonstrates that postnatal somatic gene transfer using in vivo transfection to transiently express Cas9 and a Ptch1 targeting gRNA is sufficient to induce $\mathrm{MB}$ in Trp53-knockout mice ('CRISPR-Ptch1 (TF)').

MB induction in WT animals using in utero electroporation. Homozygous deletion of Ptch1 in ventricular zone stem cells (hGFAP-Cre;Ptch $1^{f l f l}$ ) at prenatal stages induces MB with a high 
a
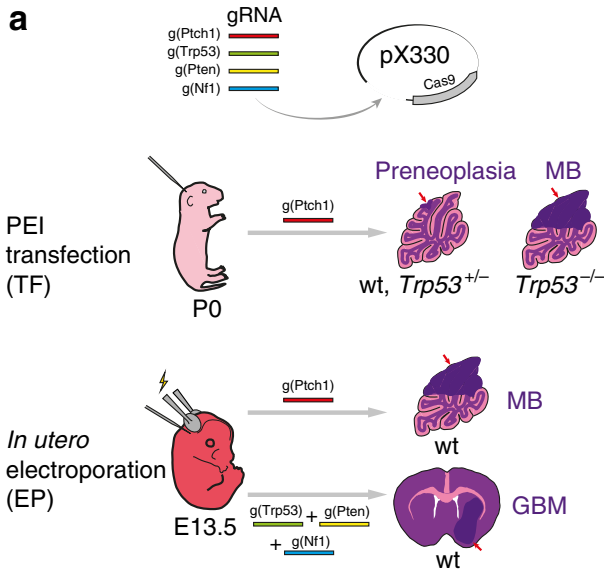

b
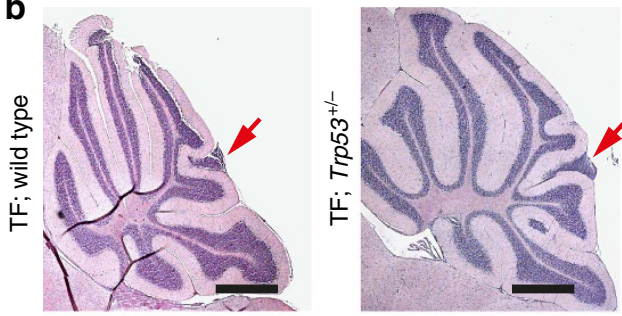

c

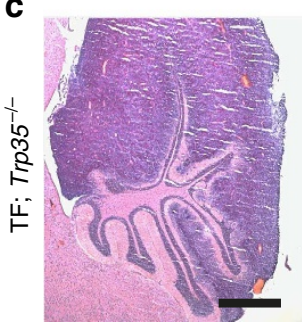

d

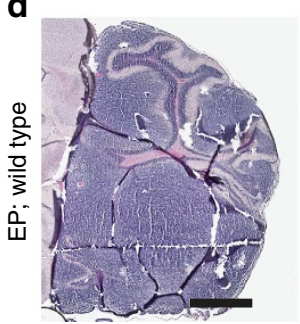

e

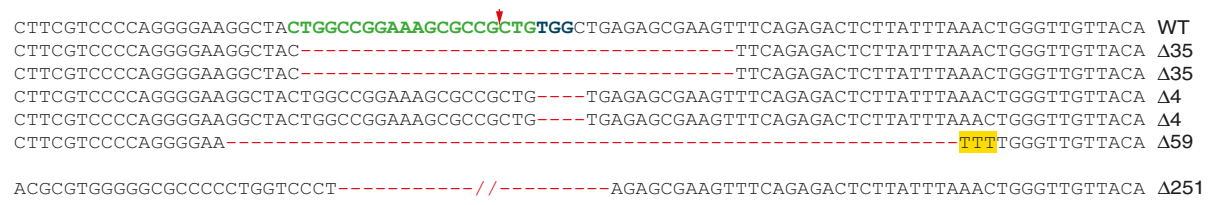

f

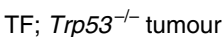

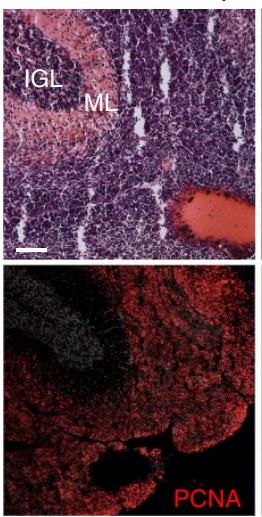

g

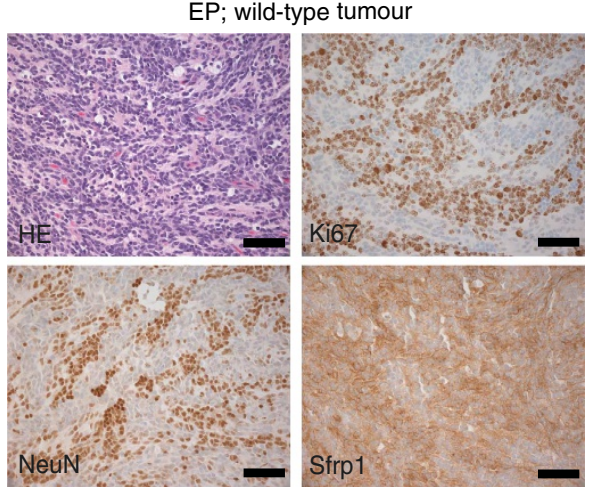

Figure 1 | CRISPR/Cas9-mediated somatic deletion of the Ptch1 locus induces medulloblastoma. (a) Cerebella of PO WT, Trp53+/ - and Trp53-/ mice were transfected with pX330 plasmids encoding Cas9 and a gRNA directed against the Ptch1 locus. In addition, plasmids were electroporated into cerebellar neuroepithelial cells of WT animals at E13.5. Furthermore, Trp53, Nf1 and Pten were targeted simultaneously by electroporation of the ventricular zone of the cerebrum to induce GBM formation. (b) Small neoplastic lesions found in WT $(n=1 / 14)$ and Trp53-/+ $(n=1 / 11)$ gPtch1/Cas9-transfected animals. The small lesions (arrows) only encompass one cerebellar folium. Scale bars, $200 \mu \mathrm{m}$. (c) H\&E staining of a cerebellar tumour induced by transfection of a Trp53-/- animal with gPtch1.1/Cas9. Scale bar, $200 \mu \mathrm{m}$. (d) Sagittal section of a WT mouse cerebellum 6 weeks after in utero EP of gPtch1.1/Cas9 showing a large tumour. Scale bars, $200 \mu \mathrm{m}$. (e) The targeted locus was amplified from tumour tissue. The PCR products were ligated into a cloning vector and sequenced via Sanger sequencing ( $n=6$ clones). Deletions were found in all (6/6) sequences and are displayed as red dashes. Nucleotide exchanges are highlighted in yellow. The gRNA target region is displayed in green, the $3^{\prime}$-PAM sequence in blue. The Cas 9 cleavage site is indicated by a red arrowhead. (f) H\&E and IF staining of cryostat sections of fully developed tumours indicate stronger proliferation (PCNA) and fewer postmitotic cells ( $\left(\mathrm{p} 27^{\mathrm{Kip} 1}\right.$ ). Tumours stained positive for the granule cell marker Atoh1. Scale bars, $100 \mu \mathrm{m}$. (g) Histological (H\&E) features and immunohistochemical detection of selected marker proteins (NeuN, Ki67/Mib1 and Sfrp1) of a representative medulloblastoma generated by somatic Ptch1 disruption. Tumours show densely packed small tumour cells with round to oval, hyperchromatic nuclei and scant cytoplasm, occasionally cells formed neuroblastic rosette-like structures. Mitotic activity was prominent but areas of necrosis were absent. The tumours invaded into the adjacent cerebellar cortex and grew within the meninges along the cerebellar surface (HE). Tumours are immunopositive for NeuN in clusters of more differentiated and less proliferative cells (NeuN). These NeuN-positive cell clusters were intermingled with clusters of NeuN-negative but strongly Mib1-positive proliferative tumour cells (Ki67). Staining for Sfrp1 is homogeneously positive. Scale bars, $50 \mu \mathrm{m}$.

penetrance and short latency ${ }^{16,17}$. As $g P t c h 1 / \operatorname{Cas} 9$ can potentially induce bi-allelic disruption of the Ptch1 gene in target cells, we sought to enhance MB genesis in WT animals by using in utero electroporation with our targeting vector at embryonic day 13.5 (E13.5), when GNPs are born in the upper rhombic lip.
To identify the cell types targeted, we used immunofluorescence staining of embryonic brains 2 days after electroporation with a GFP-encoding plasmid. Immunofluorescence revealed that $\mathrm{Pax} 2^{+}$cells (cerebellar inhibitory interneuron progenitors) and Sox $2^{+}$cells (neural stem cells) in the ventricular zone as well 


\begin{tabular}{|c|c|c|c|c|}
\hline gRNA & Method & $\begin{array}{c}\text { Genotype } \\
\text { Trp53 }\end{array}$ & $\begin{array}{l}\text { Tumour } \\
\text { incidence }\end{array}$ & $\begin{array}{c}\text { Killed (age in weeks) to } \\
\text { check for tumour } \\
\text { development }\end{array}$ \\
\hline gPtch1.1 & $\mathrm{TF}$ & WT & $1 / 14^{*}$ & $0 / 14$ \\
\hline gPtch1.1 & TF & $+/-$ & $1 / 8^{\star}$ & $2 / 8(6 w), 2 / 8(8 w)$ \\
\hline gPtch1.1 & TF & $-1-$ & $5 / 5$ & $1 / 5(8 w)$ \\
\hline gPtch1.3 & TF & WT & $0 / 0$ & $0 / 0$ \\
\hline gPtch1.3 & TF & $+1-$ & $0 / 3$ & $0 / 3$ \\
\hline gPtch1.3 & TF & $-1-$ & $3 / 5$ & $2 / 5(11 w)$ \\
\hline gNT & TF & WT & $0 / 4$ & $0 / 4$ \\
\hline gNT & TF & $+1-$ & $0 / 2$ & $2 / 2(7 w)$ \\
\hline gNT & TF & $-1-$ & $0 / 3$ & $2 / 3(7 w)$ \\
\hline gPtch1.1 & EP & WT & $7 / 7$ & $1 / 7(7 w)$ \\
\hline gPtch1.3 & EP & WT & $4 / 5$ & $0 / 5$ \\
\hline gTrp53 & EP & WT & $0 / 6$ & $0 / 6$ \\
\hline
\end{tabular}

Abbreviations: EP, tumour induced by in utero electroporation with gPtch1.1/Cas9; gNT, negative control gRNA without genomic target; gRNA, guide-RNA; TF, tumour induced by PEl-mediated transfection with gPtch1.1/Cas9; w, weeks; WT, wild type. Mice were killed after control intervals (age indicated), when presented with neurological symptoms or at 6 months of age. Mice counted as having no tumour displayed histology without pathological findings and/or did not

show neurological symptoms by 6 months of age.

${ }^{\star}$ Small neoplastic region only.

as Pax6 ${ }^{+}$GNPs in the upper rhombic lip were targeted by in vivo electroporation (Supplementary Fig. 6). We selected animals with successful gene transfer by co-electroporating a plasmid encoding a transposon-flanked luciferase gene together with a transposase gene, thereby allowing for intravital bioluminescence imaging (Supplementary Fig. 7). Of animals that were electroporated with gPtch1/Cas9 that showed luciferase signal after birth, 11/12 developed tumours within 16 weeks of age (Supplementary Fig. 5, Table 1). After dissection of the brain, a clear enlargement of the cerebellum could be observed (Supplementary Fig. 8). Post mortem analysis using $\mathrm{H} \& \mathrm{E}$ staining revealed the presence of $\mathrm{MB}$ encompassing the majority of the cerebellum ('CRISPR-Ptch1 (EP)') (Fig. 1d). As controls, we electroporated mice with a gRNA targeting Trp53 (gTrp53) together with Cas9 but none of these mice developed a tumour $(n=0 / 6$; Table 1$)$.

To verify intended deleterious Ptch1 variants induced by gPtch1/Cas9, we isolated genomic DNA from the neoplastic lesions and amplified the Ptch1 locus around the gRNA target site by PCR. Agarose gel electrophoresis showed multiple products of varying size, as compared with the normal control that yielded the expected single band (Supplementary Fig. 9). Sanger sequencing of the subcloned PCR products revealed the presence of deletions ranging from 4 to $251 \mathrm{bp}$ in size. Of note, all of these deletions resulted in a frameshift of the Ptch1 open-reading frame (Fig. 1e).

We characterized the CRISPR-Ptch1 tumours histologically in more detail to verify their phenotypic similarity to human MB. $\mathrm{H} \& \mathrm{E}$ staining revealed that the small preneoplastic lesions identified in the cerebellum of $g P t c h 1 /$ Cas9-injected WT and $\operatorname{Trp} 53^{+/-}$animals were cell-dense, circumscribed lesions and positive for the granule cell marker $\mathrm{Pax}^{28}$ and the postmitotic marker p27 ${ }^{\text {Kipl } 1}$ (Supplementary Fig. 10). CRISPR-Ptch1 tumours (TF and EP) were also highly proliferative (PCNA-positive) and positive for the granule cell marker Atoh $1^{29}$, with considerably fewer postmitotic cells (p27 ${ }^{\mathrm{Kip} 1}$ ) (Fig. 1f). This suggests that both types of lesions were derived from the granule cell lineage but that the tumours had an enhanced proliferative phenotype. Furthermore, the CRISPR-Ptch1 tumours showed a histological appearance reminiscent of nodular (desmoplastic) $\mathrm{MB}$, the prototypic histopathological variant of $\mathrm{SHH} \mathrm{MB}$ (Fig. 1g). The NeuN-positive cell clusters were intermingled with clusters of NeuN-negative but strongly with Mib1-positive proliferative tumour cells, while staining for Sfrp1, a Shh pathway target and signature gene for the human $\mathrm{SHH}$ MB subgroup ${ }^{30}$, was strongly positive. These data show that the generated CRISPR-Ptch1 tumours histologically resemble human $\mathrm{SHH} \mathrm{MB}$.

Molecular characteristics of CRISPR/Cas-induced MB. To further examine the molecular characteristics of somatically induced CRISPR-Ptch1 MBs, we isolated total RNA from six tumours (three in utero electroporation on a WT background, three postnatal transfection Trp53 ${ }^{-/}$) and performed arraybased gene expression analysis. CRISPR-Ptch1 expression profiles were compared with the respective published profiles of other murine MB models as well as normal adult mouse cerebellum and postnatal murine GNPs. Pairwise unsupervised clustering using a subset of the most variant genes (top 5\%, $n=1,076$ ) showed that both types of CRISPR-Ptch1 tumours are most similar to germline Ptch $1^{+/-}$and Atoh1-CreER;Ptch $1^{\text {flffl16 }}$ MB models. In contrast, both CRISPR-Ptch1 models are clearly distinct from normal postnatal GNPs or MB mouse models representing Wnt $(C t n n b 1 ; \operatorname{Trp} 53)^{31}$, Group $3(M y c ; \operatorname{Trp} 53)^{32,33}$ or Group4 $(M y c n)^{34}$ (Fig. 2a). In addition, in both CRISPR-Ptch1 models, a series of SHH target genes (Mycn, Gli1/2, Sfrp1, Hhip, Boc) showed an increased expression that was comparable to that observed in the other Ptch1 models and in GNPs (Fig. 2b). Of note, Ptch1, which is a Shh target gene itself, can still be detected at an appreciable expression level. However, the observed frameshift mutations are predicted to prevent expression of a functional protein as indicated by Shh pathway activation in these tumours. These data clearly demonstrate that our somatically induced MBs histologically and molecularly resemble $\mathrm{MB}$ of the relevant subgroup.

To analyse the indels generated by somatic gene transfer of gPtch1/Cas9 in greater detail, we performed targeted ultra-deep sequencing of the Ptch1 locus in three different tumours (two CRISPR-Ptch1 (EP), one CRISPR-Ptch1 (TF); Supplementary Table 1) and matched normal tissue using five different staggered amplicons (251 bp paired-end, on average $>200,000$-fold coverage per amplicon; Supplementary Data 1). In addition, to capture the full picture of genomic variants around the Ptch1 locus, we performed WGS of the same six samples (101 bp paired-end, on average 23.3-fold coverage; Supplementary Data 2). In all three MBs, non-frameshift indels as well as the WT Ptch1 locus were highly underrepresented or absent in the WGS data (Fig. 2c). This indicates that both Ptch1 alleles were disrupted in the tumours. In the targeted sequencing data, we identified 21, 22 and 34 different indels per tumour sample ( $>1 \mathrm{bp}$; represented in $>0.5 \%$ of reads in at least two different amplicons), indicating a high degree of polyclonality. The majority of sequencing reads $(>75 \%)$ were representing at most three indels per sample, though, which indicates the existence of few predominant clones (Fig. 2d). Interestingly, even indels with the highest read-score in this data set were underrepresented in the WGS data, indicating that most of the deletions induced by gPtch1/Cas9 could not be detected with amplicon-based sequencing approaches (Fig. 2e,f).

Our WGS data also facilitated a genome-wide analysis of potential off-target effects of $g P t c h 1 / \operatorname{Cas} 9$ in the aforementioned tumours. To firstly perceive a full picture of genomic variants, we applied a permissive filter setting and obtained 122,364 deletions in all of the six analysed samples in total. However, none of these mapped with the sequence of our Ptch1 gRNA even when allowing for up to three mismatches, besides the deletions in Ptch1 itself (Supplementary Data 3). When filtering for loci that were recurrently mutated in all three samples we found only Ptch1 as bona fide hit (Supplementary Data 4). To look for potential off-target effects in more detail, we filtered for loci 
a

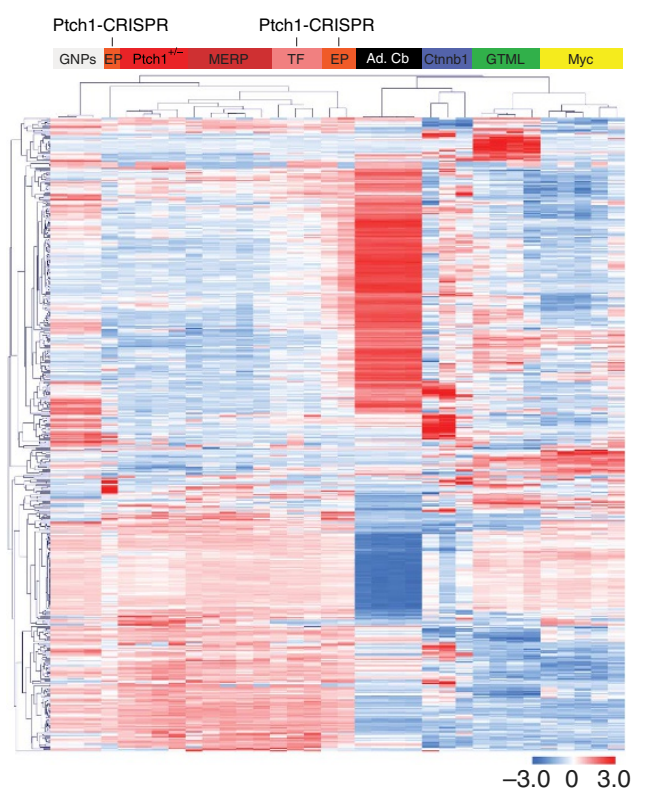

b

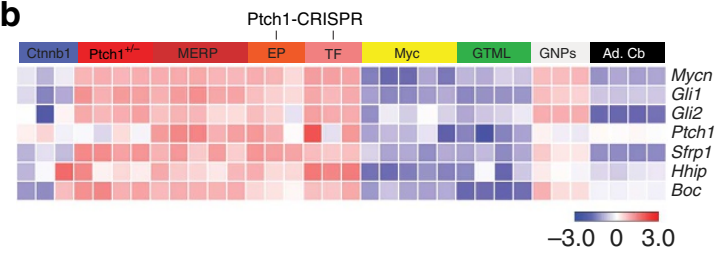

e

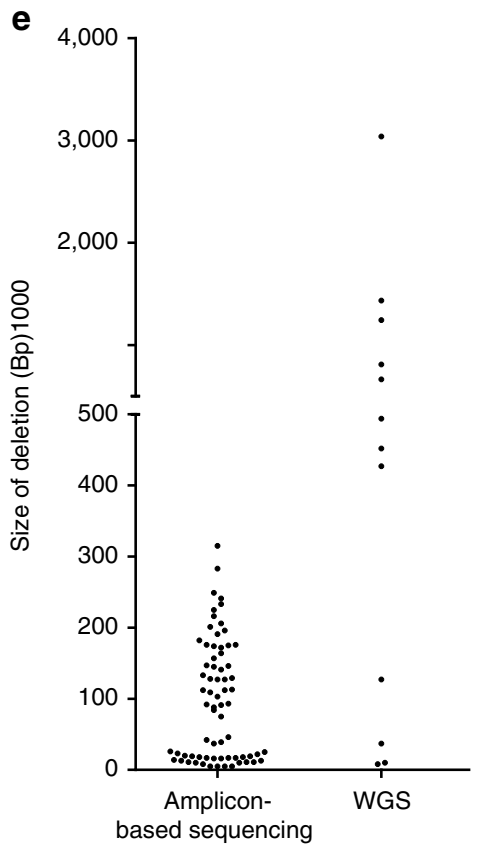

C

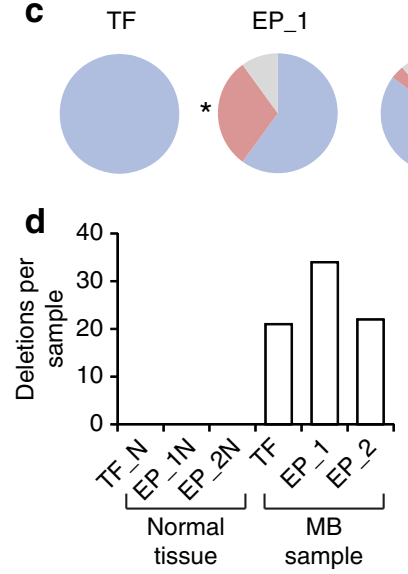

EP_2

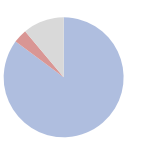

- $3 \mathrm{~N}+1 / 2 \mathrm{Bp}$

- $3 \mathrm{~N}+\mathrm{OBp}$

WT
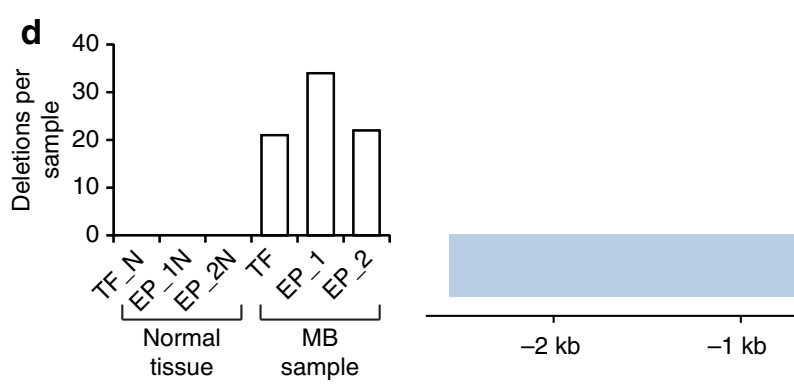

f

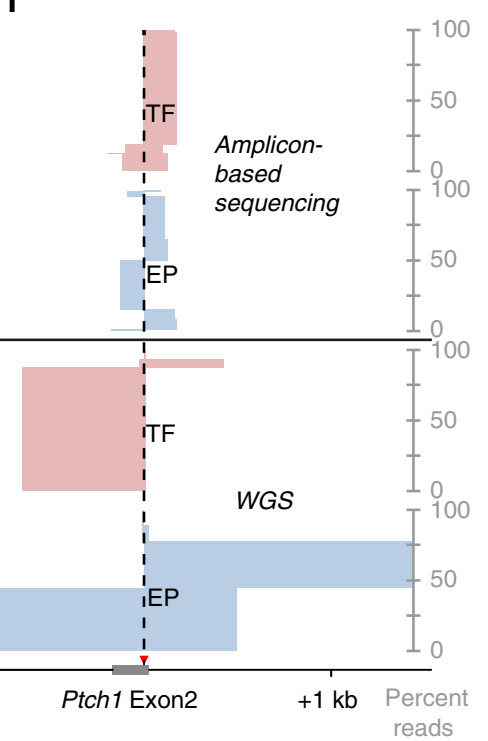

Figure 2 | Molecular characterization of CRISPR/Cas9-induced tumours. (a) Unsupervised clustering of gene expression data generated from CRISPR-Ptch1 tumours or taken from published data sets of other medulloblastoma mouse models. (b) Shh pathway target genes are upregulated in CRISPR-Ptch1 tumours. (c) Fraction of frameshift deletions in three different tumour samples. Reads covering the targeted Ptch1 locus in the WGS data set were analysed for either showing the WT allele, containing a frameshift deletion (deletion size within the exon equals $3 \mathrm{~N}+1 / 2 \mathrm{bp}$ ) or a deletion that does not result in a frameshift (deletion size equals $3 \mathrm{~N}+\mathrm{O} \mathrm{bp}$ ). The fraction indicated by an asterisk represents a single, big deletion supported by three reads, encompassing 1,435 bp. (d) Amount of different bona fide deletions found in analysed samples. The targeted Ptch1 locus of indicated CRISPR-Ptch1 tumours and matched normal tissue was amplified with five different primer pairs and analysed by deep sequencing. A deletion was counted if it ranged $>1 \mathrm{bp}$ and was represented in $>0.5 \%$ of reads in at least two different PCR products. (e) Size of deletions found in the Ptch1 locus in the targeted deep sequencing compared with the WGS data set of the three analysed PTCH1-CRISPR tumours. For the targeted deep sequencing data, a deletion was counted if it ranged $>4 \mathrm{bp}$ and was represented in $>0.5 \%$ of reads in at least two different PCR products. (f) Genomic landscape of Ptch1 deletions found in a representative CRISPR-Ptch1 (TF) (red boxes) and a CRISPR-Ptch1 (EP) (blue boxes) tumour. The targeted region within the Ptch1 locus is indicated by a red arrow head. The width of each box indicates the size of the deletion, whereas the height indicates the fraction of reads supporting this deletion. For the targeted amplicon-based deep sequencing all deletions were included that occurred in $>0.5 \%$ of the reads of the PCR product generated with the primers Seq_PTCH_1.1 and Seq_PTCH_1.2. (TF = tumour induced by PEI-mediated transfection with gPtch1.1/Cas9, EP = tumour induced by in utero electroporation with gPtch1.1/Cas9, Myc $=$ Myc T58A plus dominant-negative Trp53 or Myc WT in Trp53-null ${ }^{32,33}, \mathrm{GTML}=$ Glt1-TRE;MycN; Luciferase ${ }^{34}$, Ctnnb $=$ Blbp-

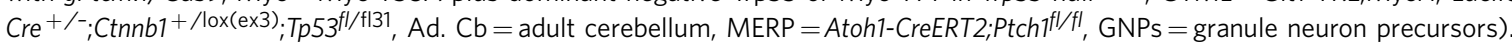


a

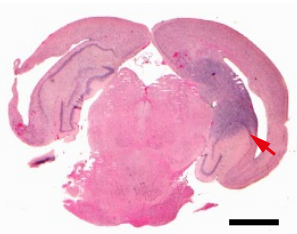

C

Nf1 1 AGAACACACATACCAAAGTCAGCACCGAGCACAAACAAGGAGTGTCTGATCAACATTT + 1 10/10

Pte

ATGGGATTTCCTGCAGAAAGACTTGAAGGTGTA-TACAGGAACAATATTGATGATGTA WT 2/10 ATGGGATTTCCTGCAGAAAGACTTGAAGGTGTAATACAGGAACAATATTGATGATGTA $\quad+1 \quad 8 / 10$

$\operatorname{Trp} 53$

CTTCTTTGTCCCATCCACAGCCATCACCTCACT-GCATGGACGATCTGTTGCTGCCCC WT 2/8 СTTCTTTGTCCCATCCACAGCCATCACСTCACTTGCATGGACGATCTGTTGCTGCCCC +1

\section{Figure 3 | Simultaneous targeting of Nf1, Pten and Trp53 loci in the}

forebrain induces GBM. (a) H\&E-stained coronal section of the brain of a WT mouse 8 weeks after in utero EP of $g$ Trp53, gPten, gNf1/Cas9 showing a tumour in one hemisphere (arrow). Scale bar, $2 \mathrm{~mm}$ (b) H\&E (left panel): cellular tumour with pleomorphic often multinucleated tumour cells and pathological vascular proliferations. H\&E (right panel): staining of the same cellular tumour displaying focal necroses with pseudopalisading of tumour cells. Tumour cells are highly proliferative as indicated by Ki67 stianing. GFAP staining showing a moderate to strong labelling of the majority of the tumour cells. Scale bars, $50 \mu \mathrm{m}$. (c) The targeted loci were PCR-amplified from tumour tissue. The PCR products were ligated into a cloning vector and sequenced via Sanger sequencing (number of clones indicated).

Nucleotide insertions are highlighted in yellow. The gRNA target regions are displayed in green, the $3^{\prime}$-PAM sequences in blue. The Cas9 cleavage sites are indicated by a red arrowhead.

displaying different deletions in one sample and again only found Ptch1 as plausible hit (Supplementary Data 5). Thus, these data indicate no detectable off-targets in this setting.

GBM induced by multiplex gene targeting. To extend the utility of this approach to other tumour entities and multiple gene targets, we sought to induce glioblastoma (GBM), the most aggressive primary brain tumour ${ }^{20}$, by simultaneously disrupting multiple TSGs using in utero electroporation. The high penetrance of MB formation demonstrated the efficiency of this method for delivering expression plasmids into the developing brain. Previously, it was shown that $h$ GFAP-Cre; $N f 1^{f /+} ; \operatorname{Tr} p 53^{f / f}$; $\mathrm{Pten}^{f /}+$ mice develop GBMs with high penetrance ${ }^{25}$. We therefore electroporated the ventricular zone of the cerebrum in E13.5 WT embryos with plasmids carrying Cas 9 and gRNAs directed against endogenous Trp53, Pten and Nf1 loci ( $g$ Trp53, gPten, gNf1). By 6 weeks of age the first animals began to show neurological symptoms (Supplementary Fig. 5). Post mortem histologic analysis revealed the presence of tumours in the forebrain with histologic features resembling GBM (Fig. 3a,b). In total $8 / 8$ animals exhibited tumours after 6-14 weeks, whereas all animals injected with either gRNA alone did not show any tumour-related symptoms (Table 2). Clonal Sanger sequencing of the three target loci revealed indels resulting in frameshifts in each of the respective genes (Fig. 3c). These data show that intracerebral somatic gene transfer of CRISPR/Cas9 can induce specific tumours via the simultaneous targeting of multiple TSGs.

\section{Discussion}

The validation of the oncogenic role of mutations identified in human tumours and the generation of animal models faithfully
Table 2 | Incidences of forebrain tumours.

\begin{tabular}{lc} 
gRNA & Tumour incidence \\
\hline gTrp53, gNf1, gPten & $8 / 8$ \\
gTrp53 & $0 / 3$ \\
gNf1 & $0 / 4$ \\
gPten & $0 / 6$ \\
\hline Abbreviation: gRNA, guide-RNA. All constructs were delivered by in utero electroporation of \\
E13.5 WT animals. Mice counted as having no tumour displayed no neurological symptoms by \\
6 months of age.
\end{tabular}

recapitulating the molecular, biological and clinical characteristics of their human counterparts are key aspects in translational oncology. Genetically engineered mouse models have contributed significantly to the better understanding of tumour biology and the preclinical testing of novel treatment approaches. However, the elaborate generation of GEMMs cannot keep pace with the ever increasing wealth of data resulting from cancer genomesequencing studies. Therefore, we set out to combine somatic gene transfer and recent developments in the field of guided nucleases to somatically disrupt TSGs in the murine brain and thereby induce different types of brain tumours. We used postnatal PEI-mediated transfection and in utero electroporation and focused on $\mathrm{MB}$, an embryonal tumour mainly occurring in young patients, and GBM, the most malignant primary astrocytic brain tumour.

Targeting Ptch1 by PEI-mediated postnatal transfection of wild-type mice or mice carrying a homozygous deletion of Trp53 evoked preneoplastic lesions or full-blown MB, respectively. This is consistent with previously published data, in which a $\operatorname{Trp} 53$ deletion can accelerate tumorigenesis in Ptch1 heterozygous $\mathrm{GEMMs}^{18}$. It had been demonstrated that bi-allelic Ptch1 deletion at embryonic stages can enhance tumorigenesis in Ptch1-driven MB models ${ }^{16,17}$. By targeting Ptch1 via in utero electroporation, we did not require additional oncogenic hits for MB induction. Therefore, our somatic gene transfer-based MB models recapitulate many features that had already been observed using genetically engineered mice.

By targeting Nf1, Pten and Trp53 simultaneously in the forebrain of E13.5 mice, we were able to induce highly aggressive tumours resembling human GBMs. Thereby, we could show that the somatic CRISPR/Cas-mediated tumour induction via in utero electroporation is compatible with multiplexing and modelling of other brain tumour entities. While multiplexing has already been shown using viral gene transfer to the liver and lung ${ }^{6,7}$, we here demonstrate that in utero electroporation can be used as a non-viral approach to target the brain, even at embryonic stages, which further complements the utility of somatic CRISPR/Cas9mediated genome editing.

We analysed the tumours induced by somatic Ptch1 deletion using amplicon-based sequencing and whole-genome sequencing (WGS). In these analyses, the wild-type allele was highly underrepresented indicating a biallelic disruption of the locus. We also investigated further characteristics of the induced indels and could show that many of the deletions we found were larger than $500 \mathrm{bp}(n=6 / 13$, Fig. 2e,f). Deletions of this size have scarcely been described as a result of CRISPR/Cas9-induced double-strand breaks so far ${ }^{2,3,35,36}$, which might partially owe to the limitations of amplicon-based sequencing methods that have been predominantly used in these studies. Detected insertions were much smaller in size $(<10 \mathrm{bp})$. As we show here, WGS can improve the detectable resolution of variants induced by CRISPR/Cas that might aid downstream data interpretation in similarly focused studies. Compared with the big deletions we found in the Ptch1 locus of CRISPR-PTCH1 tumours, the small 
insertions identified in the induced GBMs hint towards a very variable repair of CRISPR-induced double-strand breaks in vivo, potentially specific for the respective cell type or genomic locus. However, further studies will be needed to elucidate the underlying repair mechanisms.

The WGS data of the CRISPR-PTCH1 tumours facilitated us to investigate the occurrence of recurrent off-target indels. With the applied coverage and filters, no recurrent off-target deletions could be identified. Although we cannot exclude the well described ${ }^{37-40}$ Cas 9 off-target activity in general, no clonal enrichment of the respective indels could be detected in this setting.

Taken together, we demonstrate that somatic gene transfer of CRISPR plasmids encoding Cas9 and gRNAs directed against single or multiple TSGs is suitable to induce distinct brain tumour entities. Furthermore, these tumours recapitulate the phenotype of the corresponding germline-based animal models and do not carry detectable off-target deletions. Thus, this approach represents a fast and versatile method to investigate larger sets of candidate TSGs in vivo not only for brain tumours but also for other cancers.

\section{Methods}

Vector construction. Oligonucleotides coding for guide RNAs that target the second exon of the Ptch1 locus were constructed and cloned into pX330 (Addgene) according to the original online protocol of the Zhang lab (http://www.genomeengineering.org/crispr/wp-content/uploads/2014/05/CRISPR-Reagent-DescriptionRev20140509.pdf). In this study, the following 20 bp sequences were targeted: Ptch1.1 5'-CTGGCCGGAAAGCGCCGCTG-3', Ptch1.2 5'-TCAGAGACTCTTA TTTAAAC-3', Ptch1.3 5'-GTTGTGGGTCTCCTCATATT-3', Nf1 5'-AGTCAG CACCGAGCACAACA- $3^{\prime}$, Pten $5^{\prime}$-AAAGACTTGAAGGTGTATAC- $3^{\prime}$ and Trp53 $5^{\prime}$-ACAGCCATCACCTCACTGCA-3' . For control experiments, a gRNA targeting the following $20 \mathrm{bp}$ sequence was designed: $5^{\prime}$-GCGACCAATACGCGAACGTC- $3^{\prime}$. The sequence does not map to the most recent mouse genome assembly (mm10) without base change or when changing any one or two positions, and maps three times when changing any three positions (analysed using SeqMap v1.0.13 (ref. 41)). For luciferase imaging of P0-transfected mice, the vector pT2C-LucPGK-SB100X was used. To control for successful electroporation the plasmids pT2K IRESluciferase and pCAGGS-T2TP ${ }^{43}$ were co-electroporated. For the construction of pT2K IRES-luciferase the gene encoding IRES-luciferase was inserted into EcoRI and XhoI sites of pT2K CAGGS ${ }^{43}$.

Surveyor assay. A total of $2 \times 10^{5} \mathrm{NIH} 3 \mathrm{~T} 3$ cells (obtained from ATCC, authenticated as murine and mycoplasma free) were seeded in six-well dishes containing $2 \mathrm{ml}$ IMDM medium (Life Technologies; supplemented with FCS, penicillin/streptavidin and glutamine) per well 1 day before transfection. Lipofectamine 2,000 (Life Technologies) was used to transfect cells with $3 \mu \mathrm{g}$ Cas9/gRNA expression vector according to the manufacturer's instructions. DNA was isolated with the QiaAMP DNA Mini Kit (Qiagen) at 3 days post transfection. The respective locus was amplified using the primers Survey_PTCH_fwd 5'-GGA TGGTCCTGGTTCCAGTC-3' and Survey500_PTCH_rev $5^{\prime}$-CCCGAGTAGAT TACAGCGCG- ${ }^{\prime}$ with PRECISOR polymerase (Biocat) in GC buffer (Biocat). Heterodimerization and digestion with SURVEYOR nuclease were performed with the SURVEYOR Mutation Detection Kit (Transgenomic) according to the manufacturer's instructions. Cleavage products were separated on a $2 \%$ agarose gel and stained with ethidium bromide for $10 \mathrm{~min}$. Images were captured with the Gel Doc system (Bio-Rad). Band intensities were quantified via ImageJ. Gene modification levels were calculated using the following equation ${ }^{44} \%$ genes modified $=\left(1-(1-\text { fraction cleaved })^{0.5}\right) \times 100$.

Animal studies. For in vivo transfection plasmid vectors were purified using the EndoFree Plasmid Maxi Kit (Qiagen). A mix of plasmid DNA and in vivo-jetPEI transfection reagent (Polyplus-transfection) was prepared according to the manufacturer's instructions. P0 C57BL/6N mice (male and female) were anaesthetised with $2 \%$ isoflurane and medially injected at lambda: -3.6 and $\mathrm{D} / \mathrm{V}$ : -0.7 with $1 \mu \mathrm{l}$ of either DNA transfection reagent mix or trypan blue using a 75 RN Neuros Syringe and a 33-gauge needle (Hamilton). For in utero electroporation, surgical operation and electroporation-based gene transfer was performed as follows. After injection of DNA plasmids $\left(1 \mu \mathrm{l}, 2.5 \mathrm{gl}^{-1}\right.$ in PBS) into the fourth ventricle (4th V.) or cerebral ventricular zone (VZ) of E13.5 $\mathrm{Crl}$ :CD1(ICR) embryos, electric square pulses were delivered laterally using forceps-like electrodes ( $32 \mathrm{mV}$ (4th V.) or $35 \mathrm{mV}$ (VZ), 50ms-on, 950ms-off, 5 pulses). The operated embryos were allowed to survive until being born and were subjected to bioluminescence imaging of luciferase activity. All animal experiments have been performed according to animal welfare regulations and have been approved by the responsible authorities (Regierungspräsidium Karlsruhe, approval numbers G-98/13; G-176/13; G-209/14).

Luciferase imaging. For mice that were co-transfected with luciferase-encoding vector pT2C-LucPGK-SB100X at P0, imaging was performed 2 days post transfection. Electroporated mice were monitored in the first postnatal week. Luciferase signals were captured using the Xenogen IVIS-100 Imaging System. Mice were anaesthetized with $2 \%$ isoflurane and injected with $3 \mathrm{mg}$ (P7) or $200 \mu \mathrm{g}$ (P2) of StayBrite D-Luciferin (Biocat) intraperitoneally 5-7 min before the imaging. Images were acquired and radiance (photons persec percm ${ }^{2}$ persteradian) was determined within regions of interest (mouse head). Only neonates whose brain carried luciferase signals were selected for further analysis.

Histological analysis. For histological and immunohistochemical analyses, brains from treated mice were fixed in $4 \%$ phosphate-buffered formaldehyde, dehydrated in a STP 120 spin tissue processor (Thermo Scientific) and embedded in paraffin. Sections ( $5 \mu \mathrm{m}$ thickness) were cut and mounted onto glass slides, deparaffinized in xylene and rehydrated in a descending series of alcohols, then rinsed in distilled water and stained. Haematoxylin-eosin (HE) staining was carried out according to routine laboratory protocols. For immunohistochemical stainings, enzyme-induced antigen retrieval (for anti-GFAP) using pepsin $(1 \%$ in $0.01 \mathrm{M} \mathrm{HCl})$ or heat-induced antigen retrieval (all other antibodies) using a $1 \times$ target retrieval solution (Dako) was performed. Primary antibodies used were rabbit anti-GFAP (Dako Z0334, 1:1,000), rabbit anti-Ki67 (CellMarque, Rocklin, CA, 275R-14, 1:200), mouse antiNeuN (Merck Millipore, Billerica, MA, MAB377, 1:200) and rabbit anti-SFRP1 (Abcam, ab4193, 1:200). Secondary antibodies were diluted 1:500 (anti-mouse, VectorLab, BA-2,000 or anti-rabbit, VectorLab, BA-1,000) and staining was visualized using the avidin-biotin peroxidase system (VectorLab) and freshly prepared diaminobenzidine as chromogen (Dako). Slides were counterstained with haematoxylin, dehydrated and mounted.

Immunofluorescence studies. Cerebella of mice were formalin-fixed and paraffin-embedded ( $8 \mu \mathrm{m}$ sections; see above). Alternatively, brains were fixed with $4 \%$ PFA in PBS for $2.5 \mathrm{~h}$ on ice, followed by cryoprotection with $30 \%$ sucrose in PBS overnight. Fixed tissues were embedded in O.C.T Compound (Sakura), frozen on dry ice and cryopreserved at $-80^{\circ} \mathrm{C}$ for cryosections ( $12 \mu \mathrm{m}$ thickness). For staining with an anti-PCNA antibody, sections were subjected to heat antigen retrieval with $10 \mathrm{mM}$ citrate buffer for $30 \mathrm{~min}$. After blocking with $10 \%$ normal donkey serum in PBS-T (0.1\% Triton X-100 in PBS) for $30 \mathrm{~min}$ at room temperature, sections were incubated with primary antibody overnight at $4{ }^{\circ} \mathrm{C}$. Incubation with secondary antibody was performed for $30 \mathrm{~min}$ at room temperature. Subsequently, sections were mounted using the ProLong Gold antifade reagent (Lifetechnologies). The antibodies used for IHC were anti-Pax6 (1:1,000, Covance, PRB-278P), anti-PCNA (1:1,000, Merck Millipore, NA03), anti-GFP (1:1,000, Abcam, ab13970), anti-GFP (1:1,000, Cell Signaling, 2,555), anti-Pax2 (1:1,000, Invitrogen, 71-6000), anti-Calbindin (1:1,000, Millipore, AB1778), anti-Sox2 (1:500, Santa Cruz, sc-17320), anti-p27 (1:1,000, BD Biosciences, 610242) and anti-Atoh1 (1:1,000, Muguruma et al. $\left.{ }^{42}\right)$.

Sanger sequencing. Tumour tissue or normal brain tissue was scraped from H\&E-stained sections and the DNA was isolated using the DNeasy Blood \& Tissue Kit (Qiagen). The respective loci were amplified using the primers Seq_PTCH_2.1 $5^{\prime}$-GCACCCCAAGTCTCATTCAG-3' and Seq_PTCH_2.2 5'-GTCTCGAGATT AGCTGCCTTT-3', Seq_Pten $1.15^{\prime}$-GCCTCAGTCGCGTATTCTG- $3^{\prime}$ and Seq_Pten_1.2 5'-CATCCAGTGACGCATCCAG-3', Seq_P53_1.1 5'-CAGGGT CTCAGAAGTTTGAGG- $3^{\prime}$ and Seq_P53_1.2 $5^{\prime}$-GCATTGAAAGGTCACAC GAAAG-3' $3^{\prime}$ or Seq_Nf1_1.1 $5^{\prime}$-GGAAAACACTGGATAGAAGATTTG-3' and Seq_Nf1_1.2 5'-GCTTCAACGGGAATAAAAACCTG-3'. The PCR products were separated on a $2 \%$ agarose gel, stained with ethidium bromide for $10 \mathrm{~min}$ and analysed with the Gel Doc system (Bio-Rad). Subsequently PCR-products of tumor tissue were cloned into pJET1.2 (Thermo Scientific). Plasmids of 6-10 colonies per analysed locus were subjected to sequencing with an ABI PRISM 7900HT Sequence Detection System (Applied Biosystems).

Targeted deep sequencing. DNA from five frozen whole cerebella and three tumours was isolated using the DNeasy Blood \& Tissue Kit (Qiagen). As controls, DNA from corresponding fresh frozen mouse tails was isolated via standard phenol/chloroform extraction. The Ptch1 locus of each sample was amplified with the following five consecutively shifted primer pairs; Seq_PTCH_1.1 5'-CTCAC TGATTTACAACCAAGGC-3 $3^{\prime}$ and Seq_PTCH_1.2 $5^{\prime}$-CATCAAACACAGTAA AGGGAAGG-3'; Seq_PTCH_2.1 5'-GCACCCCAAGTCTCATTCAG-3' and Seq_PTCH_2.2 5'-GTCTCGAGATTAGCTGCCTTT-3'; Seq_PTCH_3.1 5'-CAC ACTGCTGTCCAGAGGG-3' and Seq_PTCH_3.2 $5^{\prime}$-ACTGGCCAGCCATGCA AAC-3'; Seq_PTCH_4.1 5' -CCTGGTCCCTAGAGTACAG-3' ${ }^{\prime}$ and Seq_PTCH_4.2 $5^{\prime}$-CGCCTGATCGCTTACCTTC-3' ${ }^{\prime}$; Seq_PTCH_5.1 5' -GGTTCCAGTCCGAG GGAG-3' and Seq_PTCH_5.2 5'-CAAGGGCTTCTCGTTGGCTA-3'. To control for a successful PCR, $1 / 10$ of PCR products were analysed on a $2 \%$ agarose gel. 
For each sample, the remainder of the 5 PCR products was pooled and purified with the QIAquick PCR Purification Kit (Qiagen). After library preparation, barcoded sequencing was performed using the Illumina MiSeq platform, yielding over 1.2 million $251 \mathrm{bp}$ paired-end reads per sample. Subsequently, reads were sorted into amplicons according to the presence of the primer sequences at the beginning of both ends, not allowing for any mismatches. Both ends were merged using Pear v0.9.4 (ref. 45) and the following non-default parameter: Pvalue 0.0001 . Resulting fragments of the same sequence were combined, and fragments occurring $<100$ times were removed. If multiple fragments of the same length per sample and amplicon were present, the sequence occurring most frequently was retained as the predominant sequence. For non-reference fragments insertions and deletions were annotated in detail. Indels were called if present in at least two amplicons and making up for at least $0.5 \%$ of the reads. Indel length is given as the length of the affected reference sequence minus the length of the alternative sequence.

Whole-genome sequencing. DNA of the same tumour and control samples were used for WGS. After library preparation, sequencing was performed using the Illumina HiSeq platform, yielding on average 330 million $101 \mathrm{bp}$ paired-end reads per sample. Sequences were aligned to the $\mathrm{mm} 10$ reference genome retrieved via the UCSC browser using the 'mem' algorithm of bwa version 0.7.10 [Li (2013) arXiv:1303.3997] and default settings. Alignments were coordinate-sorted and merged using Picard. For the downstream analysis of indels, both ends were treated individually and only reads containing at least two mapping stretches (CIGAR code M) separated by stretches of insertions or deletions, as well as split reads mapping to the same chromosome, were retained. Indels were extracted using a custom R-script, making use of the GenomicAlignments BioConductor package. Individual reads containing indels were retained whether each mapping stretch was at least $25 \mathrm{bp}$ in size, and at least $95 \mathrm{bp}$ were mapped in total. Individual reads indicating the same indel were combined, while filtering putative PCR duplicates. On the basis of results from the targeted sequencing, resulting deletions were retained if being between $4 \mathrm{bp}$ and $100 \mathrm{~kb}$ in size. In addition, both start and end position of deletions were required to not overlap start or end positions of deletions in the combined control samples (extended by $5 \mathrm{bp}$ ), or share $>80 \%$ identity with known polymorphic indels in mouse ${ }^{46}$. Indels were further filtered if start or end positions (extended by $5 \mathrm{bp}$ ) overlapped annotated simple repeats (RepeatMasker, retrieved via the UCSC browser) or regions with a combined coverage larger than 250 in the control samples. All WGS data were re-aligned to a reference set comprising the resulting sequences of the indels identified by targeted sequencing or indels affecting the Ptch1 locus in the genome-wide WGS analysis.

Gene expression analysis. Published gene expression array profiles for murine Myc-driven ${ }^{32}$ and Wnt-driven ${ }^{47}$ MBs were accessed from the Gene Expression Omnibus under the accession numbers GSE33199 and GSE24628, respectively. Total RNA was extracted from murine cerebellar tumours, non-neoplastic GNPs and normal adult mouse cerebellum, and hybridized to Affymetrix Mouse Genome 4302.0 arrays at the Genomics and Proteomics Core Facility of the DKFZ according to the manufacturer's instructions. Affymetrix CEL files were processed using Affymetrix Expression Console (v1.3.1) and normalized signal intensities were generated using the RMA algorithm. Two-dimensional unsupervised hierarchical clustering (Pearson Correlation, average linkage) of tumour and normal gene expression profiles was performed using the top 5\% of high s.d. genes $(n=1,076)$ across the samples. Clustering results and expression heat maps were generated using the TM4 MultiExperiment Viewer ${ }^{48}$.

\section{References}

1. Garraway, L. A. \& Lander, E. S. Lessons from the cancer genome. Cell 153, 17-37 (2013).

2. Cong, L. et al. Multiplex genome engineering using CRISPR/Cas systems. Science 339, 819-823 (2013).

3. Mali, P. et al. RNA-guided human genome engineering via Cas9. Science 339, 823-826 (2013).

4. Jinek, M. et al. A programmable dual-RNA-guided DNA endonuclease in adaptive bacterial immunity. Science 337, 816-821 (2012).

5. Valerie, K. \& Povirk, L. F. Regulation and mechanisms of mammalian double-strand break repair. Oncogene 22, 5792-5812 (2003).

6. Platt, R. J. et al. CRISPR-Cas9 knockin mice for genome editing and cancer modeling. Cell 159, 440-455 (2014).

7. Xue, W. et al. CRISPR-mediated direct mutation of cancer genes in the mouse liver. Nature 514, 380-384 (2014).

8. Abdallah, B. et al. A powerful nonviral vector for in vivo gene transfer into the adult mammalian brain: polyethylenimine. Hum. Gene Ther. 7, 1947-1954 (1996).

9. Saito, T. In vivo electroporation in the embryonic mouse central nervous system. Nat. Protoc. 1, 1552-1558 (2006).

10. Taylor, M. D. et al. Molecular subgroups of medulloblastoma: the current consensus. Acta. Neuropathol. 123, 465-472 (2012).

11. Ostrom, Q. T. et al. CBTRUS statistical report: Primary brain and central nervous system tumors diagnosed in the United States in 2006-2010. Neuro-oncol. 15(Suppl 2): 1-56 (2013)
12. Northcott, P. A. et al. Medulloblastomics: the end of the beginning. Nat. Rev. Cancer 12, 818-834 (2012)

13. Wechsler-Reya, R. J. \& Scott, M. P. Control of neuronal precursor proliferation in the cerebellum by Sonic Hedgehog. Neuron 22, 103-114 (1999).

14. Goodrich, L. V., Milenkovic, L., Higgins, K. M. \& Scott, M. P. Altered neural cell fates and medulloblastoma in mouse patched mutants. Science 277, 1109-1113 (1997).

15. Wetmore, C., Eberhart, D. E. \& Curran, T. The normal patched allele is expressed in medulloblastomas from mice with heterozygous germ-line mutation of patched. Cancer Res. 60, 2239-2246 (2000).

16. Yang, Z. J. et al. Medulloblastoma can be initiated by deletion of Patched in lineage-restricted progenitors or stem cells. Cancer Cell 14, 135-145 (2008).

17. Schuller, U. et al. Acquisition of granule neuron precursor identity is a critical determinant of progenitor cell competence to form Shh-induced medulloblastoma. Cancer Cell 14, 123-134 (2008).

18. Wetmore, C., Eberhart, D. E. \& Curran, T. Loss of p53 but not ARF accelerates medulloblastoma in mice heterozygous for patched. Cancer Res. 61, 513-516 (2001).

19. Kool, M. et al. Genome sequencing of SHH medulloblastoma predicts genotype-related response to smoothened inhibition. Cancer Cell 25, 393-405 (2014).

20. Gonzales, M. The 2000 World Health Organization classification of tumours of the nervous system. J. Clin. Neurosci. 8, 1-3 (2001).

21. Zong, H., Parada, L. F. \& Baker, S. J. Cell of origin for malignant gliomas and its implication in therapeutic development. Cold Spring Harb. Perspect. Biol. 7, a020610 (2015).

22. Alcantara Llaguno, S. et al. Malignant astrocytomas originate from neural stem/ progenitor cells in a somatic tumor suppressor mouse model. Cancer Cell 15, 45-56 (2009).

23. Verhaak, R. G. et al. Integrated genomic analysis identifies clinically relevant subtypes of glioblastoma characterized by abnormalities in PDGFRA, IDH1, EGFR, and NF1. Cancer Cell 17, 98-110 (2010).

24. Brennan, C. W. et al. The somatic genomic landscape of glioblastoma. Cell 155, 462-477 (2013)

25. Chen, J. et al. A restricted cell population propagates glioblastoma growth after chemotherapy. Nature 488, 522-526 (2012).

26. Zhu, Y. et al. Early inactivation of p53 tumor suppressor gene cooperating with NF1 loss induces malignant astrocytoma. Cancer Cell 8, 119-130 (2005).

27. Oliver, T. G. et al. Loss of patched and disruption of granule cell development in a pre-neoplastic stage of medulloblastoma. Development 132, 2425-2439 (2005).

28. Duan, D., Fu, Y., Paxinos, G. \& Watson, C. Spatiotemporal expression patterns of Pax6 in the brain of embryonic, newborn, and adult mice. Brain Struct. Funct. 218, 353-372 (2013).

29. Akazawa, C., Ishibashi, M., Shimizu, C., Nakanishi, S. \& Kageyama, R. A mammalian helix-loop-helix factor structurally related to the product of Drosophila proneural gene atonal is a positive transcriptional regulator expressed in the developing nervous system. J. Biol. Chem. 270, 8730-8738 (1995).

30. Northcott, P. A. et al. Medulloblastoma comprises four distinct molecular variants. J. Clin. Oncol. 29, 1408-1414 (2011).

31. Gibson, P. et al. Subtypes of medulloblastoma have distinct developmental origins. Nature 468, 1095-1099 (2010).

32. Kawauchi, D. et al. A mouse model of the most aggressive subgroup of human medulloblastoma. Cancer Cell 21, 168-180 (2012).

33. Pei, Y. et al. An animal model of MYC-driven medulloblastoma. Cancer Cell 21, 155-167 (2012).

34. Swartling, F. J. et al. Pleiotropic role for MYCN in medulloblastoma. Genes Dev 24, 1059-1072 (2010).

35. Cho, S. W., Kim, S., Kim, J. M. \& Kim, J. S. Targeted genome engineering in human cells with the Cas9 RNA-guided endonuclease. Nat. Biotechnol. 31, 230-232 (2013).

36. Jinek, M. et al. RNA-programmed genome editing in human cells. eLife 2, e00471 (2013).

37. Fu, Y. et al. High-frequency off-target mutagenesis induced by CRISPR-Cas nucleases in human cells. Nat. Biotechnol. 31, 822-826 (2013).

38. Hsu, P. D. et al. DNA targeting specificity of RNA-guided Cas9 nucleases. Nat. Biotechnol. 31, 827-832 (2013).

39. Pattanayak, V. et al. High-throughput profiling of off-target DNA cleavage reveals RNA-programmed Cas9 nuclease specificity. Nat. Biotechnol. 31, 839-843 (2013).

40. Cho, S. W. et al. Analysis of off-target effects of CRISPR/Cas-derived RNA-guided endonucleases and nickases. Genome Res. 24, 132-141 (2014).

41. Jiang, H. \& Wong, W. H. SeqMap: mapping massive amount of oligonucleotides to the genome. Bioinformatics 24, 2395-2396 (2008).

42. Muguruma, K. et al. Ontogeny-recapitulating generation and tissue integration of ES cell-derived Purkinje cells. Nat. Neurosci. 13, 1171-1180 (2010). 
43. Sato, Y. et al. Stable integration and conditional expression of electroporated transgenes in chicken embryos. Dev. Biol. 305, 616-624 (2007).

44. Guschin, D. Y. et al. A rapid and general assay for monitoring endogenous gene modification. Methods Mol. Biol. 649, 247-256 (2010).

45. Zhang, J., Kobert, K., Flouri, T. \& Stamatakis, A. PEAR: a fast and accurate Illumina Paired-End reAd mergeR. Bioinformatics 30, 614-620 (2014).

46. Keane, T. M. et al. Mouse genomic variation and its effect on phenotypes and gene regulation. Nature 477, 289-294 (2011).

47. Robinson, G. et al. Novel mutations target distinct subgroups of medulloblastoma. Nature 488, 43-48 (2012).

48. Saeed, A. I. et al. TM4: a free, open-source system for microarray data management and analysis. Biotechniques 34, 374-378 (2003).

\section{Acknowledgements}

We thank the DKFZ Proteomics and Genomics Core Facility for next generation sequencing (Stephan Wolf and colleagues) and gene expression analysis

(M. Bewerunge-Hudler and colleagues), DKFZ animal facility staff and in particular B. Goedecke-Weichert for support with animal work, B. Rumpel, L. Linke and

D. Goldnik for technical support, D. Grimm for discussion, R. Wechsler-Reya and

S. Brun for discussion and providing MERP tumours, K. Muguruma for Atohl antibody as well as K. Kawakami and Y. Takahashi for Tol2-related plasmids. This work has been supported by the Helmholtz Alliance 'Preclinical Comprehensive Cancer Center' (PCCC; Grant No. HA-305). C.B.K-.T. was supported by the Max Eder program of the German Cancer Aid (Deutsche Krebshilfe). B.T. was supported by a NIH training grant T32 AI 83196-5.

\section{Author contributions}

J.G., M.Zu. and P.L. conceived the strategy. J.G., D.K., P.L. and S.M.P. supervised the project. M.Zu., D.K., K.S. and J.B. performed the experiments. D.K. performed the in utero electroporations. V.H., M.Za. and P.A.N. performed bioinformatics analyses. B.T., B.M. and D.L. provided methodological expertize. C.B.K-.T, G.R. and A.K. did the histologic analysis and neuropathologic evaluation. M.F.R. provided material and criticisms of the manuscript. J.G., M.Zu., D.K., P.L., S.M.P., P.A.N., D.T.W.J. and G.R. wrote the manuscript.

\section{Additional information}

Accession codes: Sequencing data associated with this study were deposited in the European Nucleotide Archive under accession code PRJEB6541. Gene expression profiles associated with this study were deposited in the Gene Expression Omnibus under accession code GSE58629.

Supplementary Information accompanies this paper at http://www.nature.com/ naturecommunications

Competing financial interests: The authors declare no competing financial interests.

Reprints and permission information is available online at http://npg.nature.com/ reprintsandpermissions/

How to cite this article: Zuckermann, M. et al. Somatic CRISPR/Cas9-mediated tumour suppressor disruption enables versatile brain tumour modelling. Nat. Commun. 6:7391 doi: $10.1038 /$ ncomms8391 (2015)

\section{(c) (1)}

This work is licensed under a Creative Commons Attribution 4.0 International License. The images or other third party material in this article are included in the article's Creative Commons license, unless indicated otherwise in the credit line; if the material is not included under the Creative Commons license, users will need to obtain permission from the license holder to reproduce the material. To view a copy of this license, visit http://creativecommons.org/licenses/by/4.0/ 